\title{
Trampoline Troubles: Serious Traumatic Injuries in Children from a Trampoline Park, a Case Series
}

\author{
Jeremy Smith ${ }^{1}$, Brendan Williams ${ }^{2}$, Tricia B. Swan ${ }^{1 *}$ \\ ${ }^{1}$ College of Medicine, University of Florida, Gainesville, FL, USA \\ ${ }^{2}$ Department of Orthopaedics, The Children's Hospital of Philadelphia, Philadelphia, PA, USA \\ Email: jeremysmith@ufl.edu,williamsba@email.chop.edu, ‘tfalgiani@ufl.edu
}

How to cite this paper: Smith, J., Williams, B. and Swan, T.B. (2019) Trampoline Troubles: Serious Traumatic Injuries in Children from a Trampoline Park, a Case Series. International Journal of Clinical Medicine, 10, 452-461.

https://doi.org/10.4236/ijcm.2019.109038

Received: August 30, 2019

Accepted: September 17, 2019

Published: September 20, 2019

Copyright $\odot 2019$ by author(s) and Scientific Research Publishing Inc. This work is licensed under the Creative Commons Attribution International License (CC BY 4.0).

http://creativecommons.org/licenses/by/4.0/

\begin{abstract}
Background: Trampoline use is one of the most common causes of recreational injury in children. In recent years, trampoline parks have grown in popularity and may be altering the spectrum of the trampoline-related injuries that occur. These parks create increased opportunity for injuries that appear uniquely different from accident patterns seen with home trampoline use. Recent work has suggested this may result in injuries occurring in greater frequency and with increased severity that may result in a greater need of hospital admission and procedural management by subspecialty services. Case Series Presentation: This case series presents three such examples occurring after the opening of a trampoline park in our local community: 1) A displaced forearm fracture requiring closed reduction and orthopedic follow-up; 2) An ankle fracture necessitating operative reduction and fixation; and 3) A facial injury with tooth avulsion prompting oral surgery consultation and endodontic follow-up. Conclusions: These cases demonstrate the complexity and severity of injury that can occur from trampoline park participation and should serve to increase awareness among health care providers of these risks to provide appropriate parental counseling and advocate for preventative measures.
\end{abstract}

\section{Keywords}

Trampoline, Injury, Pediatrics, Trampoline Parks, Trauma, Children

\section{Background}

Trampolines have long been a staple of childhood enjoyment and recreational 
play. However, the rising incidence of Trampoline-Related Injuries (TRIs) cannot be overlooked, accounting for over 100,000 emergency department visits in the United States annually and representing a significant portion of pediatric accidental injuries [1] [2] [3]. In the past, trampolining took place predominantly in the home with injuries occurring most often from improper landings onto or falling off the trampoline. Recently, however, we have seen a growth in the popularity of trampoline parks, changing the environment in which trampolining occurs. Instead of a single jumping surface, as seen with home trampoline use, by design, these parks place numerous jumpers and trampolines together in close proximity providing increased opportunities for collisions and falls. As one may anticipate, recent literature suggests these parks are contributing both to the rising frequency and severity of TRIs [4] [5] [6]. Unlike water parks and amusement parks, most trampoline parks are not currently regulated by a national standard. Recently, members of the International Association of Trampoline Parks attempted to develop international safety standard guidelines, however, the standards are voluntary and parks are not required to follow or adhere to the guidelines. There are not current standard regulations limiting the number, sizes or ages of jumpers or the design and placement of trampolines and other activities used in many popular trampoline parks in the United States or other countries. Most parks require participants or their parents to sign risk warnings and a waiver of liability. Currently, Arizona is the only state in the United States that has passed legislation in an attempt to regulate trampoline park safety.

Injuries sustained while trampolining most commonly occur in children and adolescents [1] [4]. Although a variety of mechanisms have been described, TRIs are most frequently due to incorrect landings or falls while on the trampoline. Other common mechanisms include falls from the trampoline mat, collisions with other jumpers, and impact from the trampoline springs or frame [4] [7] [8]. Multiple simultaneous jumpers, attempted acrobatic stunts and participants who are of varying weight and height jumping at the same time on the trampoline are the most cited risk factors for injury [4] [7] [9] [10]. As a result, younger and smaller children are at the greater risk of injury. Fractures, soft tissue injuries, dislocations, and lacerations are most commonly sustained, however, more serious and potentially fatal spine and skull injuries have been reported [4] [11] [12]. Due to this level of severity, many TRIs may require urgent and emergent medical attention.

In January of 2016, a trampoline park opened in our local community of Gainesville, Florida. Since this time, pediatric emergency medicine providers at area hospitals have noted that TRIs appear to be occurring with greater frequency and presenting with diagnoses carrying greater morbidity. In this report, we present three cases demonstrating the variety of injuries that have been cared for since the park opened. Each is unique in mechanism, pattern and treatment required, providing a good representation of the breadth and complexity of injuries that can occur with trampoline park use. 


\section{Case Series Presentation}

Case 1: A 12-year-old female with no past medical history presented to the pediatric emergency department with an arm injury occurring immediately prior to arrival at a local trampoline park. She had been jumping on a trampoline when she attempted to do a flip and landed on her left arm while outstretched. On arrival, the patient was noted to have a noticeable forearm deformity with a neurovascularly intact limb. She was provided with adequate pain and nausea control. Radiographs demonstrated a middle-third fracture of both the radius and ulna with 32 degrees of apex volar angulation (Figure 1(a)). Based on her age and the degree of angulation, the patient required conscious sedation with ketamine in the emergency department for reduction and long arm casting by the orthopedic service. Her angular deformity was reduced to 11 degrees and left without rotational malalignment (Figure 1(b)). The procedure was uncomplicated and her neurovascular status remained unchanged. The patient was discharged with follow-up in the orthopedic clinic. After 6 weeks of immobilization, she went on to fracture union with mild-but acceptable-residual angular deformity (Figure 1(c)). At her 8-week follow up visit with the orthopedic surgeon, she demonstrated no loss of elbow motion with a 20-degree reduction in pronosupination. She was ultimately released to full activities.

Case 2: A 14-year-old female with no past medical history presented to the pediatric emergency department two hours after injuring her ankle at a local trampoline park. She reported the injury occurred after an awkward landing on her left ankle, twisting it in an unknown fashion. She described pain that progressively worsened and was unable to bear weight. The limb demonstrated substantial soft tissue swelling and tenderness diffusely but was without significant limb deformity and was neurovascularly intact. The patient received pain control in the emergency department and underwent radiographs of the left ankle which identified a Salter-Harris IV fracture to the distal left tibia and a Salter-Harris II fracture to the distal left fibula on radiographic imaging (Figure 2(a)). Further imaging with a computed tomography (CT) scan identified a comminuted, minimally displaced fracture of the medial malleolus with extension into the posterior malleolus as well as a minimally displaced transverse fracture of the lateral malleolus. Based on these findings, operative intervention was warranted for restoration of the articular surface. The patient was initially splinted and sent for orthopedic outpatient follow-up for surgery. With near complete closure of her physes noted, the patient was managed with open reduction and internal fixation of the medial malleolus with two cannulated screws (Figure 2(b)). At her 12-week follow up with the orthopedic surgeon, the patient demonstrated stable alignment without hardware complication (Figure 2(c)). She has initiated full weight bearing on the ankle.

Case 3: A 17-year-old male with no past medical history presented to a free-standing emergency department with a facial injury that occurred just prior to arrival. The patient had been jumping at the local trampoline park when he 


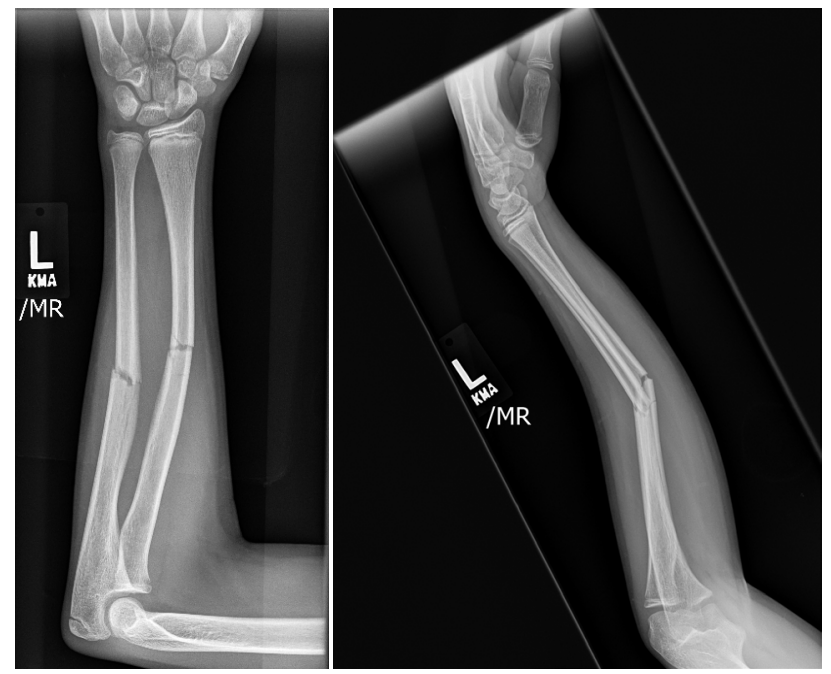

(a)

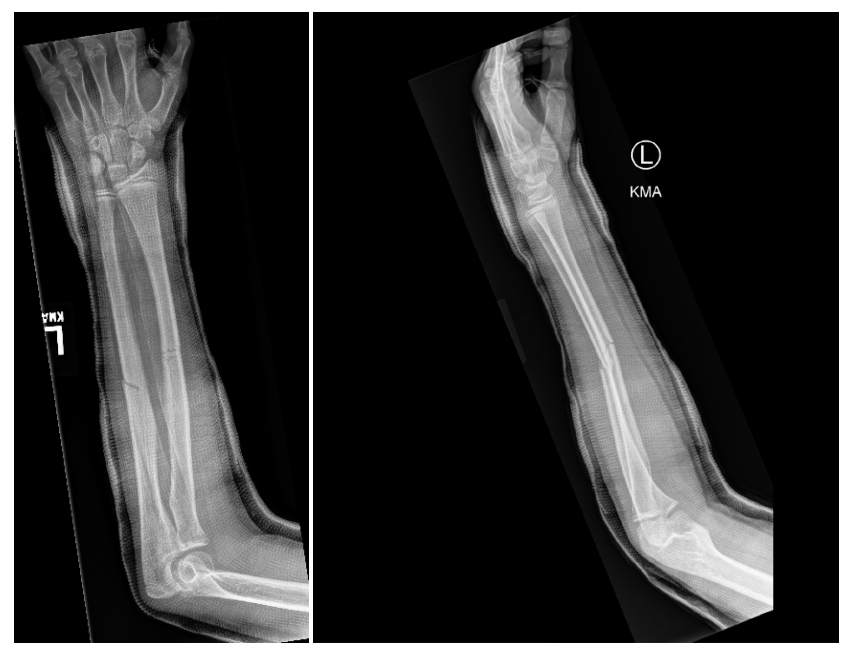

(b)

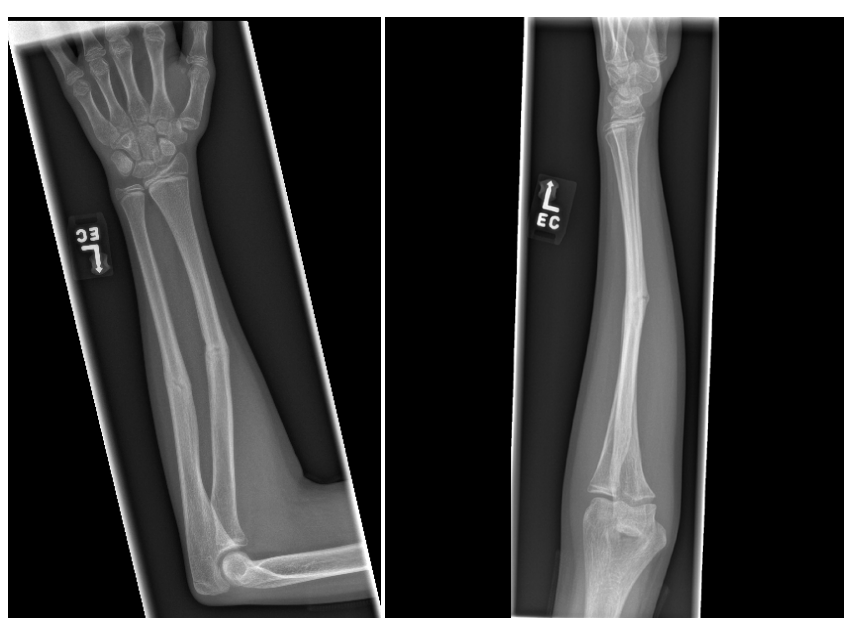

(c)

Figure 1. (a) AP and Lateral radiographs of a both bone forearm fracture with apex volar angular deformity. (b) AP and Lateral radiographs of the forearm status post reduction and casting demonstrating improved angular deformity. (c) AP and Lateral radiographs of the forearm with interval bridging callous and maintained alignment after cast removal. 


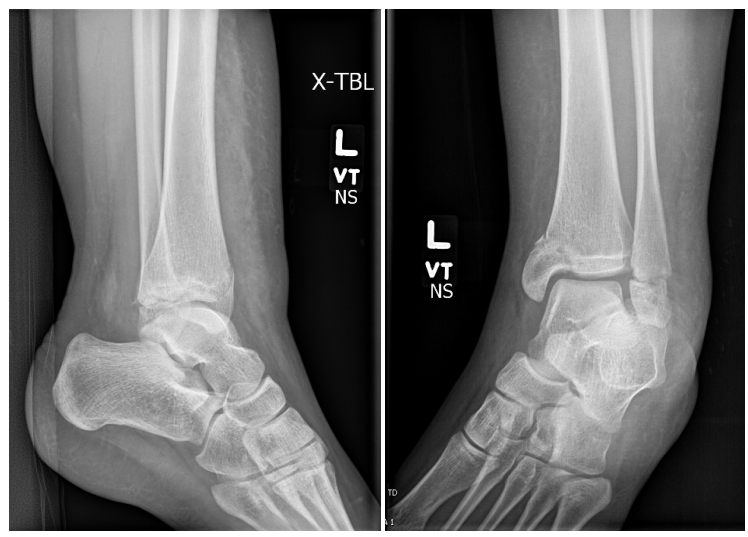

(a)

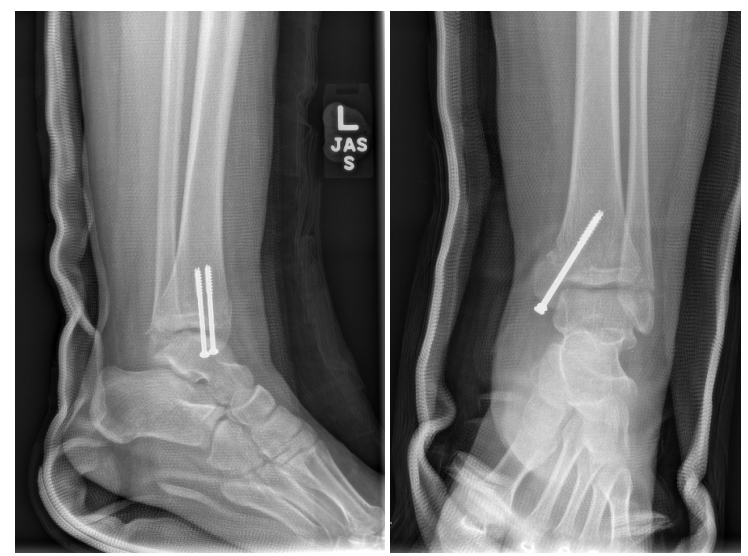

(b)

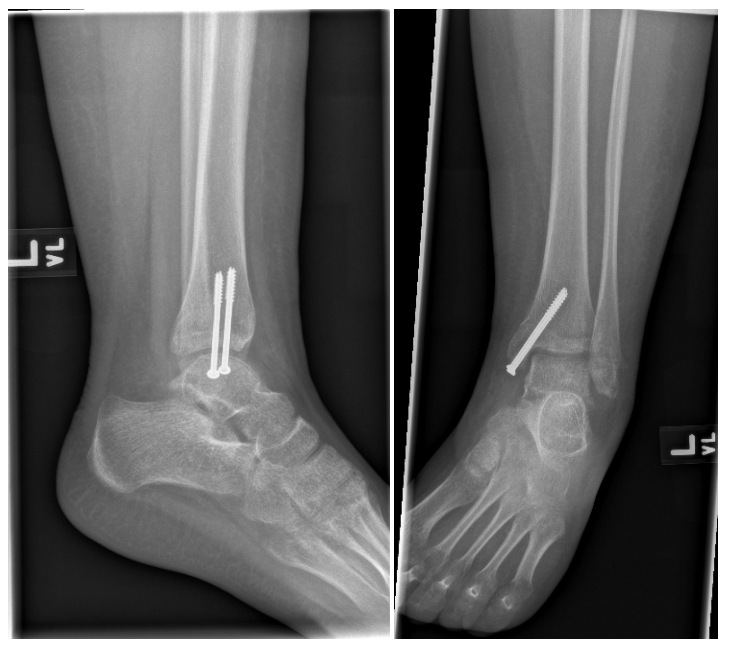

(c)

Figure 2. (a) Lateral and Mortise radiographs of the ankle in a patient nearing skeletal maturity demonstrating a minimally displaced trimalleolar ankle fracture. (b) Lateral and AP radiographs after open reduction and internal fixation of the medial malleolus. (c) Lateral and Mortise radiographs of the ankle after removal of immobilization showing fracture union with intact hardware.

landed in an uncontrolled fashion, driving his knees into his face. He was noted to have bleeding from his mouth along with oral and maxillary pain. Workup at 
the emergency department showed subluxation of tooth \#7 and \#26 with avulsion of tooth \#8, as well as a laceration to the upper gingival tissue and both lips. The patient then underwent a CT scan showing minimally displaced fractures through the alveolar ridge of the maxilla and mandible as well as a non-displaced fracture through the left maxillary buccal cortex adjacent to the root apex of tooth \#8 (Figure 3). Tooth \#8 was re-implanted by the emergency physician.

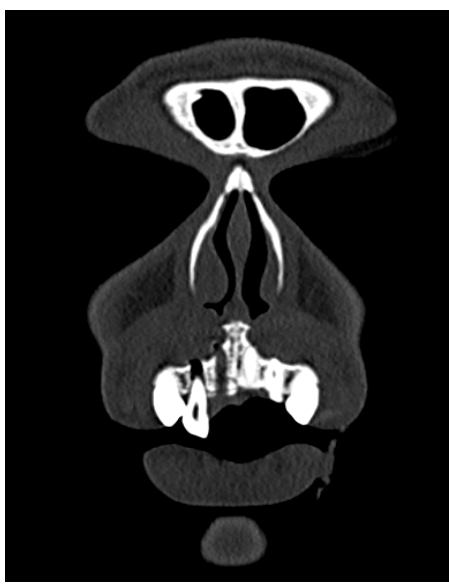

(a)

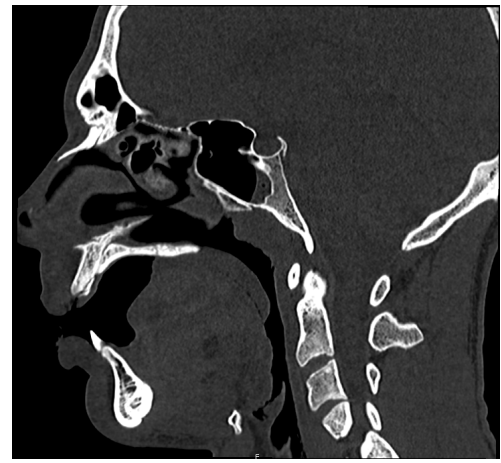

(c)

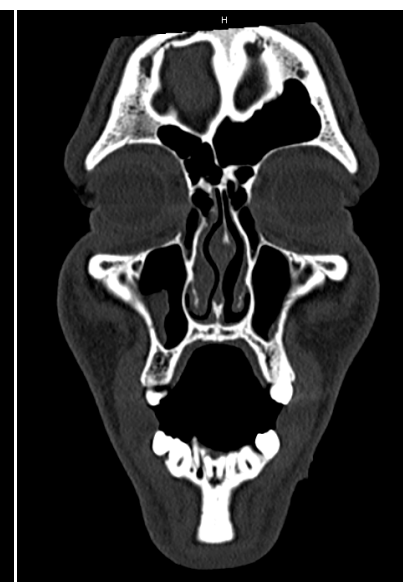

(b)

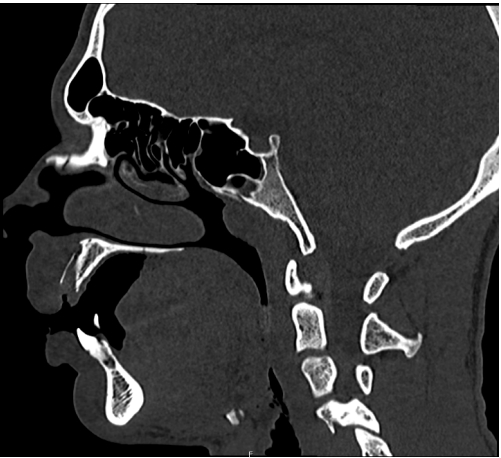

(d)

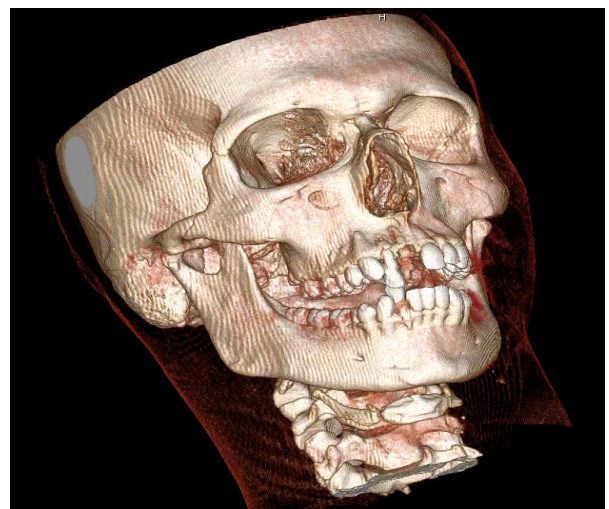

(e)

Figure 3. Selected coronal (a) and (b), sagittal (c) and (d) and three-dimensional reconstruction (e) of a maxillofacial CT scan demonstrating traumatic extraction of the right upper central incisor and subluxation of the upper and lower right lateral incisors with an associated fracture through the alveolar ridge of the maxilla and mandible. 
The patient was then transferred to a pediatric emergency department for further care. Upon arrival, he underwent oral surgery evaluation with subsequent repair of his lacerations and splinting of the teeth. Upon discharge, the patient was referred to the endodontic department for further outpatient management. 3 weeks after his initial injury he required a root canal on tooth \#8, which was done by an endodontic specialist. He continues to require frequent follow visits to the endodontist to monitor the health of his injured teeth.

\section{Discussion}

This case series highlights three unique injuries occurring at a local trampoline park to demonstrate the variety and severity of TRIs that can occur in these spaces. As seen in this case series, the injuries are variable and can be severe with long-lasting effects on the pediatric patient. Our cases highlighted 2 orthopedic injuries, one with long term range of motion deficit noted at the patient's follow up visit with the orthopedic surgeon. One case presented has required extensive dental repair after his injury and his injured teeth are still undergoing active care with and endodontist. As with home trampoline injuries, park TRIs most often result from improper landings or falls while on the trampoline. [4] [7] [8] The chance of injury increases with multiple individuals jumping simultaneously or attempting acrobatic maneuvers [4] [7] [9] [10], both of which are likely more common in a large group setting where risk-taking behavior can be greater. Our first case highlights an example of an attempted acrobatic maneuver resulting in injury. This patient required the utilization of multiple resources in the emergency department visit for radiographic diagnosis and pain control, anesthesia for procedural sedation, and orthopedic consultation for definitive management and follow-up until fracture union. The occurrence of this mechanism of injury may be reduced with improvements in safety measures and supervision of minors at parks.

Prior investigations have demonstrated that TRIs most frequently involve the musculoskeletal system, with $80 \%$ of patients requiring emergency department radiographs, and $41 \%$ requiring orthopedic procedures with anesthesia [2] [11] [13]. Results from a review of a national database by Loder et al. [13] demonstrated that $29 \%$ of TRIs sustain fractures, most commonly in the appendicular skeleton. This study also estimated that the cost of an orthopedic TRI presenting to the ED is nearly $\$ 800$ while those requiring admission or surgical intervention can cost over $\$ 7000$. Therefore, there is a definitive financial effect on both the patient and the healthcare system, adding to the substantial morbidity of trampoline park TRIs. Our second case demonstrates the extent of healthcare utilization that can be necessary following these injuries. This trimalleolar ankle fracture required an emergency department visit for diagnosis and stabilization, outpatient surgery with open reduction and internal fixation requiring general anesthesia as well as ongoing outpatient orthopedic follow-up until fracture union and return to full weight-bearing. A growing number of TRIs resulting in fractures that necessitate surgery could have a tremendous impact on healthcare spending. 
Although musculoskeletal injury is most common, a broad spectrum of injuries have been identified following trampoline accidents. These injuries can lead to extensive workups with advanced imaging, specialist consultation for management and follow-up and prolonged recovery periods. Among the nonmusculoskeletal injuries lacerations, concussions and traumatic brain injury are most commonly described. [1] [4] [7] Orofacial injuries, however, are infrequently reported. Thus our third case, a facial injury with tooth avulsion and multiple facial fractures, is illustrative of the severity of even the most unique injuries that occur. This patient required an emergency department visit with transfer to higher level of care, a CT scan, an oral surgery consultation for initial management and ongoing endodontic outpatient follow-up for definitive care.

\section{Conclusion}

With the rising popularity of trampoline parks, medical providers are expected to see a growing number of TRIs and greater utilization of subspecialty services. Although the literature regarding accidents occurring at trampoline parks is sparse, early findings suggest these facilities may be associated with increased frequency and severity of resulting injuries [4] [5] [6]. The experience of our local frontline providers would support these findings based on observations following the recent opening of a trampoline park in our community. This case series presents three such injuries occurring in pediatric patients, each requiring acute procedural intervention and extended outpatient follow-up. The current American Academy of Pediatrics (AAP) policy statement on trampoline use in children recommends against the recreational use of all trampolines, both in the home and trampoline parks. Additionally, the AAP advises that if parents allow their children to participate in trampolining that precautions must be followed to attempt to reduce injuries. Some of these precautions include restricting trampoline use to a single jumper at one time, use of appropriate padding around the trampoline and not allowing somersaults, flips or other acrobatic maneuvers while jumping [14]. Further study is warranted to discern whether or not the presence of trampoline parks increase the frequency or alter the variety of injuries occurring in a given population. These cases contribute to the growing body of literature that highlights the injuries sustained from trampoline park usage. Currently, no industry standard regulations exist for trampoline parks. As we add to our collective knowledge of such injuries, healthcare providers can advocate for standardized safety measures as well as contribute to public education and injury prevention awareness. Ultimately, these efforts will aid in advocating for measures to prevent the occurrence and reduce the severity of trampoline-related injuries among children and adolescents.

\section{Consent for Publications}

Written informed consent was obtained from the patient's guardian for publication of this case series and accompanying images. 


\section{Availability of Data and Materials}

Data sharing is not applicable to the article and no datasets were generated or analyzed during the current study.

\section{Authors' Contributions}

TS contributed to the conception and identified cases for presentation. JS, BW and TS contributed to the drafting and revision of the manuscript. All authors read and approved the final manuscript.

\section{Conflicts of Interest}

The authors declare that they have no competing interests regarding the publication of this paper.

\section{References}

[1] Linakis, J.G., Mello, M.J., Machan, J., Amanullah, S. and Palmisciano, L.M. (2007) Emergency Department Visits for Pediatric Trampoline-Related Injuries: An Update. Academic Emergency Medicine, 14, 539-544. https://doi.org/10.1111/j.1553-2712.2007.tb01826.x

[2] Rättyä, J. and Serlo, W. (2008) Using a Safety Net and Following the Safety Instructions Could Prevent Half the Paediatric Trampoline Injuries. European Journal of Pediatric Surgery, 18, 261-265. https://doi.org/10.1055/s-2008-1038534

[3] National Electronic Injury Surveillance System (NEISS). CPSC.gov. https://www.cpsc.gov/Research--Statistics/NEISS-Injury-Data

[4] Kasmire, K.E., Rogers, S.C. and Sturm, J.J. (2016) Trampoline Park and Home Trampoline Injuries. Pediatrics, 138, e20161236.

https://doi.org/10.1542/peds.2016-1236

[5] Sharwood, L.N., Adams, S., Blaszkow, T. and Eager, D. (2018) Increasing Injuries as Trampoline Parks Expand within Australia: A Call for Mandatory Standards. Aus tralian and New Zealand Journal of Public Health, 42, 153-156. https://doi.org/10.1111/1753-6405.12783

[6] Roffe, L., Pearson, S., Sharr, J. and Ardagh, M. (2018) The Effect of Trampoline Parks on Presentations to the Christchurch Emergency Department. New Zealand Medical Journal, 131, 43-53.

[7] Smith, G.A. (1998) Injuries to Children in the United States Related to Trampolines, 1990-1995: A National Epidemic. Pediatrics, 101, 406-412. https://doi.org/10.1542/peds.101.3.406

[8] Eberl, R., Schalamon, J., Singer, G., Huber, S.S., Spitzer, P. and Höllwarth, M.E. (2009) Trampoline-Related Injuries in Childhood. European Journal of Pediatrics, 168, 1171-1174. https://doi.org/10.1007/s00431-008-0897-5

[9] Hurson, C., Browne, K., Callender, O., O’Donnell, T., O’Neill, A., Moore, D.P., et al. (2007) Pediatric Trampoline Injuries. Journal of Pediatric Orthopaedics, 27, 729-732. https://doi.org/10.1097/BPO.0b013e318155ab1

[10] Klimek, P.M., Juen, D., Stranzinger, E., Wolf, R. and Slongo, T. (2013) Trampoline Related Injuries in Children: Risk Factors and Radiographic Findings. World Journal of Pediatrics, 9, 169-174. https://doi.org/10.1007/s12519-013-0416-2

[11] Furnival, R.A., Street, K.A. and Schunk, J.E. (1999) Too Many Pediatric Trampoline 
Injuries. Pediatrics, 103, e57. https://doi.org/10.1542/peds.103.5.e57

[12] Chalmers, D.J., Hume, P.A. and Wilson, B.D. (1994) Trampolines in New Zealand: a Decade of Injuries. British Journal of Sports Medicine, 28, 234-238. https://doi.org/10.1136/bjsm.28.4.234

[13] Loder, R.T., Schultz, W. and Sabatino, M. (2014) Fractures from Trampolines: Results from a National Database, 2002 to 2011. Journal of Pediatric Orthopaedics, 34, 683-690. https://doi.org/10.1097/BPO.0000000000000189

[14] Trampoline Safety in Childhood and Adolescence (2012) The American Academy of Pediatrics Policy Statement. Pediatrics, 130, 774-779.

https://doi.org/10.1542/peds.2012-2082

\section{Abbreviations}

TRI-Trampoline Related Injury;

CT-Computed Tomography;

AAP-American Academy of Pediatrics. 